\title{
Quality and safety are rarely simple
}

\section{David P Stevens}

I live on an island 70 miles from Boston. I occasionally travel to the US mainland by ferry, but more often via a small airline with nine-passenger planes that are usually flown by a solitary pilot. The pilot's preflight checklist is a small plastic card, usually reviewed in a matter of seconds-arguably, a simple task. One checklist item is to assure that the flaps are retracted, unlike jetliners that take off with flaps lowered to increase the wing surface area for lift. On a recent flight, the pilot reviewed the checklist and proceeded down the Boston runway. At an altitude of perhaps $10 \mathrm{~m}$, her right hand momentarily moved off the throttle and quickly activated the lever to fully retract the flaps. In spite of reviewing the checklist, she had initiated takeoff with the flaps down. Was this caused by momentary inattention? Boredom? Perhaps she had recently piloted larger planes. Checklists and professional autonomy are brought to mind with increasing frequency, and, as in this case, not always in reassuring settings.

\section{A COMPLEX CONTEXT FOR SIMPLE TASKS}

Given the attention that is appropriately focused on the role for complexity science in healthcare improvement, ${ }^{1}$ Liu and colleagues $^{2}$ (see page 93) seek to remind us of the simple and complicated tasks that also offer opportunities for improving healthcare quality and patient safety. Simple solutions_-such as standardised order forms and checklists-are invariably embedded in complex systems that make their implementation less straightforward than may appear on first glance. Nevertheless, their report makes the case that such forcing functions can play a role in improving timeliness of antibiotic administration for community-acquired pneumonia (CAP). ${ }^{2}$

I am not sure that the simple and complicated bits are always so easy. The message that stands out in Liu and colleagues' report is that health professionals

Correspondence to: Dr David P Stevens, Quality Literature Program, Dartmouth Institute for Health Policy and Clinical Practice, 30 Lafayette Street, Lebanon, NH 03766 USA; David.P.Stevens@Dartmouth.Edu should seek out, analyse deeply and engage in simple and complicated approaches when possible. Mathews and Pronovost remind $u^{3}$ that simple activities such as checklists, when properly applied, are wise and just obligations for healthcare providers. And yet, the pursuit of such strategies can be less than fully effective. I continue to be bemused that we persist in trying to implement the simple task of handwashing with only mixed success, even though effectiveness data for handwashing have been available since the era of Simmelweiss.

A corollary lesson appears to be that the careful identification of a precise, simple strategy tailored to a particular context may be just what is needed to override health professionals' broad and deep knowledge that otherwise compels them to insist on autonomous-but not always correct-decision making. Mills et al, for example, reported that training and education to avoid adverse drug events (ADEs) actually had a negative effect on ADEs in the absence of alerts and other forcing functions provided by a medication order entry system in the US Veterans Affairs Healthcare System. ${ }^{4}$

\section{A POSSIBLE ALTERNATIVE TO ALL-OR- NONE RULES}

In selecting antibiotic initiation time for CAP, Liu et al picked a much-debated measure that has its share of detractors. ${ }^{5}$ Yet implicit in their report ${ }^{2}$ is an important insight regarding all-or-none global practice rules such as the 4-h administration of antibiotics for CAP. Careful dissection of such global rules can lead to simple, complicated and complex options that are embedded in such rules and may direct the provider to the correct application of all or part of the rule in the appropriate patient. All-or-none is then replaced by context-driven, critical, professional judgement about what is appropriate in simple, complicated or complex ways. For example, simple therapeutic rules that are based on evidence usually trump patient preferences ${ }^{5}$; disordered, complicated patient physiology trumps simplistic therapeutic rules; and, generally, complex social and emotional contexts such as the hypothetical example of the elderly patient with the do-notresuscitate preference ${ }^{2}$ trump complicated patient management decisions. In this regard, the question may be less a matter of autonomy versus a global guideline, and more a matter of adapting best-fit components of evidence-based practice to the precise context of the patient at hand.

\section{FEEDBACK PROVIDED BY TEAMS AND SYSTEMS}

Amalberti and colleagues suggested that the option to implement simple solutions requires a collaborative or team context to achieve highest levels of safety. ${ }^{6}$ Liu et al built into their hospital practice environment, real-time feedback that supplied the provider with performance information and clinical outcomes. This system-level feedback-similar to the feedback function found in high performance teamshelped identify system issues that interfered with a flexible and informed provider-patient relationship. It helped maintain an appropriate balance between autonomy and adherence to clearly defined performance measures.

I often reflect on possible explanations for what was going on that day on the Boston airport runway. One possibility is that performance of simple tasks by highly trained experts may just degrade on frequent repetition. Another possibility-apparently not applicable in this case-is that there are occasions when experts appropriately override rules in the interest of safety. ${ }^{6}$ However, I think that there is a third, straightforward explanation. An autonomous (solitary) pilot, not unlike the autonomous practitioner, must perform tasks in high-risk contexts that may just be too complicated in the absence of the feedback provided by a high-performance team. I'll wager the flaps would have been in the correct position had there simply been a copilot in the adjacent seat.

\section{Competing interests: None.}

Qual Saf Health Care 2009;18:82-83 doi:10.1136/qshc.2009.032797

\section{REFERENCES}

1. Glouberman S, Zimmerman B. Complicated and complex systems: what would successful reform of Medicare look like? Discussion paper no. 8. Saskatoon: Commission on the Future of Health Care in Canada, 2002.

2. Liu SK, Homa KA, Butterly JR, et al. Improving the simple, complicated and complex realities of community-acquired pneumonia. Oual Saf Health Care 2009;18:93-8. 
3. Mathews SC, Pronovost PJ. Physician autonomy and informed decision making. Finding the balance for patient safety and quality. JAMA 2008;300:2913-15.

4. Mills PD, Neilly J, Kinney LM, et al. Effective interventions and implementation strategies to reduce adverse drug events in the Veterans Affairs (VA) system. Qual Saf Health Care 2008;17:37-46.

5. Wachter RM, Flanders SA, Fee C, et al. Public reporting of antibiotic timing in patients with pneumonia: lessons from a flawed performance measure. Ann Intern Med 2008;149:29-32.

6. Amalberti R, Auroy Y, Berwick D, et al. Five system barriers to achieving ultrasafe health care. Ann Intern Med 2005;142:756-64.

\title{
Market-based control: the solution to slow progress with patient safety?
}

\author{
Richard G Thomson
}

In this issue of Quality and Safety in Health Care, there is a paper that should stimulate considerable debate (see page 99); ${ }^{1}$ indeed, we have published three commentaries alongside the paper ${ }^{2-4}$ to initiate this and a further response from the authors (see page 90). ${ }^{5}$ Despite major initiatives to improve patient safety, there is a perception that attempts to improve safety have made slow progress. Hence, Coiera and Braithwaite argue for the implementation of market based control mechanisms as an incentive to promote patient safety. Their proposal is modelled on the "cap and trade" approach to creating a market in emissions trading, a key component of the Kyoto protocol that allows organisations that are successful in reducing carbon emissions to sell credits to organisations that have been less successful.

The parallel between emissions trading to improve the environment and patient safety event trading to improve healthcare safety is fascinating; each of our commentators is intrigued by the proposal. However, each of them believes that the model, while intriguing, is unlikely to be implemented or effective. Coiera and Braithwaite have responded to these commentaries with a further robust argument!

Chin and Wilkes ${ }^{2}$ are concerned that a market of this type will lead to further widening of inequalities through a variety of mechanisms including lobbying power, resource constraints, ability to pass or shift costs and gaming. If market mechanisms were to enhance inequalities, this would be unacceptable (see page 88 ). While Chin and Wilkes believe that measurement of patient safety and holding

Correspondence to: Professor Richard G Thomson, Department of Epidemiology and Public Health, School of Health Sciences, Medical School, Framlington Place,

Newcastle upon Tyne NE2 4HH, UK;

richard.thomson@newcastle.ac.uk healthcare organisations accountable in a fair manner, consistent with Coiera and Braithwaite's proposals, are important, neither is unique to market solutions.

Donaldson $^{3}$ expresses a fundamental concern-he argues that healthcare is not a public good in the same way as the environment (see page 87). He also points out that the introduction of quasimarkets in healthcare has been largely unsuccessful in addressing issues of quality and safety. Instead he calls for more explicit and better developed methods to determine priorities for investment in constrained healthcare systems. He also, quite rightly, raises the question as to whether the emissions trading model has yet shown itself to be effectiveindeed, Coiera and Braithwaite themselves accept that it is too early to evaluate that.

Meltzer ${ }^{4}$ points out that incentives for patients and payers to avoid errors through competitive market forces already exist, in contrast to carbon emissions prior to trading, thus making the argument less compelling (see page 86). He highlights the challenge of measurement and of how an appropriate level of adverse events might be set. He also believes that such a system is likely to increase healthcare costs. Meltzer flags up one element of the proposal that he describes as compelling; that is the underlying assumption that some level of harm is appropriate or acceptable because reducing harm is costly.

In addition to the concerns expressed in these commentaries, I believe there are several other issues that need to be considered before pursuing an MBC approach. First, this approach is very top down; it appears to ignore the importance of engagement of healthcare staff in improving safety. The mantra of "first do no harm" is embedded within the culture of most healthcare professionals, and when patient safety incidents occur, they are rarely due to negligence or intended actions but largely reflect the inevitabilities of human error and the inadequacies of systems. A top-down model such as that proposed here is likely to provoke resistance among professional groups.

It is also likely to provoke resistance among patients and the public. What level of acceptability would this engender within the public domain, particularly given Meltzer's comments that an underlying implication is that there is a level of acceptable harm? One of the challenges to patient safety has been the fact that the value placed upon harm produced by healthcare is often quite different to the value placed upon injury or ill health arising de novo. Fundamentally, patients access healthcare with the expectation that it will make them better and find the concept that it might make them worse very difficult to understand.

Another issue of relevance is the complexity of healthcare. The issue of carbon emission is arguably much more straightforward in both its measurement and its aetiology than harm caused by healthcare. This complexity in healthcare may explain why some of the methods of quality and safety improvement that have been effective in industrial settings are more difficult to apply in healthcare. Market-based control is likely to be similar in this respect.

A key problem, also flagged up by our commentators, is that of measurement. We know that incident reporting significantly under-reports for a variety of reasons. Equally, there is evidence to suggest that those organisations that report more incidents have a better and more effective safety culture. ${ }^{6}$ Any market-based mechanism that penalised higher rates of incidents would have the potential effect of switching off the tap of reporting, upon which much safety improvement depends. The approach would be replete with perverse incentives. Furthermore, the use of measures of safety or quality from routine information systems, such as the AHRO indicators suggested by Coiera and Braithwaite, would need to take account of the fact that routine data quality and completeness are hugely variable, not only across 